\title{
Overexpression of microRNA-1288 in oesophageal squamous cell carcinoma
}

Vinod Gopalan $^{\dagger 1}$, Farhadul Islam ${ }^{\dagger 1}$, Suja Pillai ${ }^{1}$, Johnny Cheuk-On Tang ${ }^{2}$, Daniel King-

Hung Tong ${ }^{3}$, Simon Law ${ }^{3}$, Kwok-Wah Chan ${ }^{4}$, Alfred King-Yin Lam ${ }^{1}$

${ }^{1}$ Cancer Molecular Pathology, School of Medicine and Menzies Health Institute Queensland, Griffith University, Gold Coast, Australia

${ }^{2}$ State Key Laboratory of Chirosciences, Lo Ka Chung Centre for Natural Anti-cancer Drug Development, Department of Applied Biology and Chemical Technology, the Hong Kong Polytechnic University

${ }^{3}$ Department of Surgery, Li Ka Shing Faculty of Medicine, The University of Hong Kong, Queen Mary Hospital

${ }^{4}$ Department of Pathology, Li Ka Shing Faculty of Medicine, The University of Hong Kong, Queen Mary Hospital

${ }^{\dagger}$ Equal to first authors

Running head: MicroRNA-1288 regulation in oesophageal cancer

Correspondence to: Professor Alfred Lam, Head of Pathology, Griffith Medical School, Gold Coast Campus, Gold Coast QLD 4222, Australia. a.lam@griffith.edu.au

Telephone +61 $756780718 \quad$ Fax +61 756780708

\section{Acknowledgements}

The authors would like to thank Griffith University for the support of the scholarship for HDR students. In addition, we would like thank Professor Gopesh Srivastava for his gifts by providing some of the cell lines.

Conflict of interest: The authors have no conflict of interest.

Key-words: miR-1288; oesophageal; squamous cell carcinoma; FOXO1 


\begin{abstract}
Purpose: This study aims to examine the expression profiles $m i R-1288$ in oesophageal squamous cell carcinoma (ESCC). The cellular implications and target interactions of ESCC cells following miR-1288 overexpression was also examined.
\end{abstract}

Methods: In total, 120 oesophageal tissues (90 primary ESCCs and 30 non-neoplastic tissues) were recruited for miR-1288 expression analysis using qRT-PCR. An exogenous $m i R-1288$ mimic and its inhibitor were used to explore the in-vitro effects of $m i R-1288$ on ESCC cells by performing cell proliferation, colony formation, cell invasion and migration assays. Localisation and modulatory changes of various $m i R-1288$ regulated proteins such as FOXO1, p53, TAB3, BCL2 and kRAS was examined using immunofluorescence and western blot.

Results: Overexpression of miR-1288 was more often noted in ESCC tissues when compared to non-neoplastic oesophageal tissues. High expression was often noted in high grade carcinomas and with metastases. Patients with high levels of miR-1288 expression showed a slightly better survival compared to patients with low miR-1288 levels. Furthermore, overexpression of miR-1288 showed increased cell proliferation and colony formation, improved cell migration and enhanced cell invasion properties in ESCC cells. In addition, miR-1288 overexpression in ESCC cells showed repression of cytoplasmic tumour suppressor FOXO1 protein expression. Inversely, inhibition of miR-1288 expression exhibited remarkable upregulation of FOXO1 protein, while expressions of other tested proteins remain unchanged.

Conclusions: Up regulation of $m i R-1288$ expression in ESCC tissues and $m i R-1288$ induced oncogenic features of ESCC cells in- vitro indicates the oncogenic roles of miR-1288 in ESCCs. Overexpression of miR-1288 play a key role in the pathogenesis of ESCCs and its modulation may have potential therapeutic value in patients with ESCC. 


\section{Introduction}

Oesophageal cancer has been reported to be the ninth most common malignancy worldwide and is composed of different histological subtypes with diverse cellular and molecular bases [1-4]. Recent studies have proven that small non coding RNAs known as microRNAs (miRNAs) play a significant role in the regulation of gene expressions in cancer cell differentiation, cell cycle progression, apoptosis, migration and metastasis [5-9]. Emerging data suggests miRNAs has the potential to act as a key regulator in the pathogenesis and progression of oesophageal carcinomas by targeting various target proteins [10].

MicroRNA-1288 (miR-1288) is located in 17p11.2 and has been reported to have roles in the pathogenesis of ovarian and colorectal cancers [9, 11, 12]. Slattery et al. has noted the association of miR-1288 expression in colorectal cancers with $\mathrm{CpG}$ island methylator phenotype positive (CIMP) and TP53 mutation using a miRNA microarray [11]. Another study on ovarian cancer has reported a predominant loss of heterozygosity in miR-1288 containing region [12]. Our most recent study has found that $m i R-1288$ can enhance cell proliferation and can alter major cell cycle events in colon cancer cells [9]. In addition, the expression of miR-1288 was correlated with various clinicopathological parameters in colorectal cancers such as tumour site, pathological staging and patient survival [9]. Currently, there is no evidence of involvement of $m i R-1288$ mediated in pathogenesis in oesophageal squamous cell carcinomas. Also targets of $m i R-1288$ have never been investigated. This study aimed to examine the in-vitro effects, target identification and clinicopathological significance of $m i R-1288$ expression in oesophageal squamous cell carcinoma. 


\section{Materials and methods}

\subsection{Selection of patients}

The patients who were chosen for this study had surgical resection for primary oesophageal squamous cell carcinoma (ESCC). The surgically resected tissues were obtained as formalin fixed (10\%) paraffin embedded tissues. Patients who underwent pre-operative chemo and radiotherapy were not included in this study. Patients were recruited with no selection bias. Ethic approval was obtained from Griffith University (MED/19/08/HREC). For each selected samples, tissues were sectioned using a microtome and stained by haematoxylin and eosin staining for histological analysis with an anatomical pathologist (AKL).

\subsection{Clinicopathological characteristics}

The histological grades and staging of selected ESCCs were assessed using World Health Organization's classification of tumours of the digestive system [13]. Patient's age, gender as well as site, size and pathological staging of ESCC were recorded systematically. After review, 90 patients (77 men; 13 women) with resections of primary ESCC were recruited. Thirty non-neoplastic tissues from oesophagus were also recruited to act as control populations. The mean age of these patients was 63 years (ranging from 39 to 83). The ESCCs comprised of 35\% ( $n=32 / 90)$ well differentiated, 46\% (n=41/90) moderately differentiated, and 19\% $(n=17 / 90)$ poorly differentiated. Eleven percent $(n=10 / 90)$ of the tumours were in located in the upper oesophagus, $49 \%(n=44 / 90)$ were in the middle oesophagus and $40 \%(n=36 / 90)$ was in the lower oesophagus. The size of the tumours ranged from $5 \mathrm{~mm}$ to $120 \mathrm{~mm}$ (mean $50 \mathrm{~mm}$ ). Majority of ESCCs patients (73\%, n=66/90) included in this study were advanced stages (stage III and stage IV) cancers. In addition, 
approximately 78\% (70/90) of the ESSC patients had lymph node metastasis at the time of surgery. Also, 10 ESCC patients were reported with distant metastasis at presentation.

The follow-up period was defined as the interval between the date of surgery for ESCC and the date of death or closing date of the study. The actuarial survival rate of the patients was calculated from the date of surgical resection of the ESCC to the date of death or last follow-up.

\subsection{Cell lines}

Two oesophageal cancer cell lines, KYSE-520 (ACC 371; oesophageal squamous cell carcinoma) and KYSE-70 (ACC 363; oesophageal squamous cell carcinoma) purchased from Leibniz Institute DSMZ (German collection of microorganisms and cell cultures) were used. Also, a non-neoplastic human cell lines HACAT (human keratinocyte from skin) was recruited as control. All the cell lines were maintained according to the manufacturer's guidelines.

\subsection{Extraction of miRNA and cDNA conversion}

Tissue blocks from the selected samples were sectioned and miRNA extraction was performed as previous reported [5, 8-9]. Briefly, the miRNA extraction from tissues and cell lines were performed with Qiagen miRNeasy FFPE Kits (Qiagen Pty. Ltd., Hilden, NRW, Germany) and miRNeasy Mini kit (Qiagen) respectively. Purity of miRNA was obtained by checking the optical density (OD) 260/280 ratio using a nanodrop spectrophotometer. The purified miRNAs were converted to cDNA using miScript Reverse Transcription kit (Qiagen) according to the manufacturer's instructions. Prior to the PCR experiments, each cDNA sample was diluted to $1.5 \mathrm{ng} / \mu \mathrm{l}$. 


\subsection{Quantitative Real-Time Polymerase Reaction (qRT-PCR)}

Expression changes of miR-1288 in ESCC tissues and different ESCC cell lines were examined as previously reported [9]. Briefly, a qRT-PCR was performed in a total volume of $20 \mu \mathrm{l}$ reaction mixture containing $10 \mu \mathrm{l}$ QuantiTech SYBER green PCR master mix (Qiagen), $1 \mu \mathrm{l}$ miScript universal primer, $1 \mu \mathrm{lmiScript}$ primer (miR-1288) assay (Qiagen), $2 \mu 1 \mathrm{cDNA}$ $(1.5 \mathrm{ng} / \mu \mathrm{l})$ template and $6 \mu \mathrm{l}$ Rase-free water. The amplification efficiencies of the reactions were normalised to that of a ubiquitous control gene RNU6B RNA (Hs_RNU6B_2 miScript Primer Assay; Qiagen). Primer sequences and thermal profiles were same as previously published [9].

Expression levels of miR-1288 were analysed as in our previous study [5, 8-9]. Fold change of miR-1288 expression was calculated using $2^{-[\text {delta][delta]Ct }}$ method by comparing the expression levels in the control tissues /cells. Inverse ratio of miR1288 versus RNU6B (control miRNA) [expression ratio] was used to represent the miRNA expression levels in different colorectal tissues and cell lines. A fold change of more than 2 was considered as high miR-1288 expression. A fold change of less than 1 was considered as low miR-1288 expression.

\subsection{Transfection of oesophageal cancer cells with miR-1288 mimic and inhibitor}

Oesophageal cancer cells (KYSE-520 and KYSE-70) were cultured approximately at $2 \times 10^{4}$ cells $/ \mathrm{cm}^{2}$ into 24 wells plate in the recommended media. After 24 hours of initial seeding, cells were transfected with miR-1288 mimic (Qiagen) (KYSE-520 $0^{+m i R-1288}$ and KYSE-70 ${ }^{+m i R-1288}$ ) at $10 \mathrm{nM}$ concentrations and with inhibitor (Qiagen) (KYSE-520 ${ }^{+m i R-1288-I n}$ and KYSE-70 ${ }^{+m i R-1288-I n}$ ) at 5nM concentrations according to the protocol published earlier [9]. Briefly, $3 \mu 1$ of transfection reagent, Hiperfect (Qiagen), was added to the miRNA mimic/inhibitor and incubate for 5 minutes at room temperature to form the complexes. 
These complexes were used to transfect the cell and transfection efficiencies were measured at 24 and 48 hours of transfection by measuring miR-1288 expression. Cells treated with scramble miRNA (KYSE-520 ${ }^{\text {miRScr }}$ and KYSE-70 ${ }^{\text {miRScr }}$ ) and transfection reagent (Hiperfect) alone (KYSE-520 $0^{\text {wildtype }}$ and KYSE-70 ${ }^{\text {wildtype }}$ ) were used as controls in this study.

\subsection{Cell proliferation assay}

To examine the effect of miR-1288 mimic transfection on proliferation of ESCC cell lines (KYSE-520 and KYSE-70), a cell counting kit-8 (CCK-8) (Sigma-Aldrich, St Louis, MO, USA) was used. Both the cell lines were first seeded in a flat-bottom 96-well plate at 1 $\times 10^{4}$ cells/well. After 24 hours of initial seeding, cells were transfected with miR-1288 mimic, inhibitor and scramble miRNA as previously described [9]. Cell proliferation was determined on days 0 to 3 with CCK-8 following manufacturer instructions.

\subsection{Colony formation assay}

To determine the effect of $m i R-1288$ overexpression on clonogenic capacity, $\sim 400$ cells were seeded in 6-well plates with complete medium. The cells (KYSE-520 and KYSE70) were then transfected with miR-1288 mimic, inhibitor and scramble control miRNA and cultured at $37^{\circ} \mathrm{C}$ in $5 \% \mathrm{CO}_{2}$ and saturation humidity. After 14 days, when microscopic clones were noted in the plate, growing of the cells was stopped. Then, the media was discarded and cells washed with phosphate buffered saline (PBS). Then, the cells were fixed with 70\% cold ethanol for 15 minutes at room temperature. Afterwards, the clones were stained with crystal violet $(0.5 \%)$ for 2 hours at room temperature and washed with tap water. Finally, after being air-dried, images of the plates were taken and clone formation rates were calculated. 


\subsection{Invasion assay}

The CultreCoat ${ }^{\circledR} 96$ well basement membrane extract (BME)-coated cell invasion assay (Trevigen, Inc. Gaithersburg, MD 20877, USA) kit was used to investigate the overexpression effect of miR-1288 mimic on in vitro cell penetration/invasion to a barrier, consisting of basement membrane components. In brief, KYSE-520 and KYSE-70 cells were cultured to $80 \%$ confluence and then passaged to a serum-free medium for 24 hours. These starved cells were collected and $50 \mu \mathrm{l}\left(1 \times 10^{6} / \mathrm{ml}\right)$ of cell suspension was added to each well of 96 wells top chamber. Transfection complex (miR-1288 mimic/inhibitor and Hiperfect) was then added to the top chamber to transfect the cells. Complete media was added to the bottom chamber and then incubated at $37^{\circ} \mathrm{C}$ in $\mathrm{CO}_{2}$ incubator for 48 hours. After incubation, $100 \mu \mathrm{l}$ cell dissociation solution/Calcein AM was added to the bottom chamber. Calcein AM was internalized and intracellular esterase cleaves it to produce Calcein (a bright fluorophore) and this fluorescence was used to quantitate the number of invasive cells.

\subsection{Western blot analysis}

Western blot analysis was performed to identify the downstream protein interactions of miR-1288. ESCC (KYSE-520 and KYSE-70) cells treated with miR-1288 mimic, inhibitor and scramble control miRNA and un-transfected (wildtype) groups were used. Total proteins from these cells were extracted with a lysis buffer (Bio-Rad, Gladesville, NSW, Australia). Subsequently, the proteins were quantitated by absorbance spectrometry. Afterwards, $30 \mu \mathrm{g}$ of total protein was separated by 15\% SDS-PAGE (Bio-Rad) and transferred to nitrocellulose membranes using Trans-Blot ${ }^{\circledR}$ Turbo transfer system (Bio-Rad). The membrane was blocked with 5\% non-fat milk powder for 2 hours at room temperature. Then the membrane was incubated with anti-rabbit FOXO1, p53 monoclonal antibody (Cell signalling Technology Inc., Danvers, MA 01923, USA) (at 1:1000), anti-mouse KRAS monoclonal antibody (Santa 
Cruz, Texas, USA) (at 1:500), anti-rabbit BCL2 polyclonal antibody (Santa Cruz) (at 1:200) and anti-rabbit TAB3 monoclonal antibody (Abcam, Melbourne,VIC, Australia) (at 1:1000) and anti-mouse monoclonal $\beta$-actin antibody (Santa Cruz) (1:2000) overnight at $4^{\circ} \mathrm{C}$. Membranes were then incubated with secondary antibody (Santa Cruz) (1:5000) at room temperature for 2 hours. FOXO1, p53, KRAS, BCL2, TAB3 and $\beta$-actin protein bands were developed and detected with a chemiluminscence HRP detection kit (Bio-Rad, USA). Images were taken with the ChemiDoc MP Imaging system (Bio-rad). Expression of proteins were quantified and normalized to $\beta$-actin with Image $\mathrm{J} 1.48$ software.

\subsection{Immunofluorescence}

To visualize localisation and the miR-1288 mediated downregulation of FOXO1 protein, immunofluorescence analysis was performed. Cells were seeded on cover slips at a density of $\sim 10,000 / \mathrm{cm}^{2}$ in 12 well plates. Transfection with miR-1288 mimic and control miRNA were performed and were incubated at $37^{\circ} \mathrm{C}$ for 24 hours in $\mathrm{CO}_{2}$ incubator. The cells were then fixed with $70 \%$ cold ethanol for 30 minutes. Afterwards, cells were blocked with 5\% BSA (in PBS) for one hour at room temperature. Cells were then washed with PBS and were incubated with anti-rabbit monoclonal FOXO1 antibody $(1: 100)$ at $4^{\circ} \mathrm{C}$ overnight. This was followed by 2 hours of incubation with secondary antibody labelled with fluorescein isothiocyanate (FITC) fluorophore (Sigma-aldrich) at room temperature. Finally, the cover slips were mounted on glass slides with mounting media containing DAPI (nuclear stain), sealed with nail polish and then observed under a confocal microscope (Nikon A1R+, Nikon Inc., Tokyo, Japan). 


\subsection{Statistical analysis}

All clinical, pathological, follow-up and miRNA expression changes were computerized. Statistical analysis was performed using the Statistical Package for Social Sciences for Windows (version 22.0, IBM SPSS Inc., New York, USA). Chi-square test or likelihood ratio was used for categorical variables. Pearson correlation test was used for continuous variables. Independent t-test and ANOVA was performed for the analysis of continuous variables in categories. Survival analysis was tested using Kaplan-Meier method. Significance level of the tests was taken at $\mathrm{p}<0.05$. 


\section{Results}

\subsection{Altered expression levels of $m i R-1288$ in ESCC tissues and cell lines}

Compared to non-neoplastic control tissues, 63\% (n=57/90) of the ESCC patients showed high expression of miR-1288 expression and remaining 37\% ESCC patients showed downregulation of $m i R-1288$. The expression level of $m i R-1288$ was noted to be increased in oesophageal cancer cell line (KYSE-520 and KYSE-70) in comparison to non-neoplastic human keratinocytes (HACAT) (Figure 1). The relative expression (ratio of expression) of $m i R-1288$ in HACAT was $0.714 \pm 0.21$ whereas, the ratios of expressions were $0.87 \pm 0.23$ and 0.88 \pm 0.174 in KYSE-520 and KYSE-70 cells respectively (Figure 1).

\subsection{Clinicopathological correlation and impact of patient survival}

Overall, high miR-1288 expression levels were associated with clinical aggressiveness of ESCC. Among the ESCC with lymph node metastasis $(\mathrm{n}=70)$, high $m i R-1288$ expression was more often noted (64\%). A similar trend of $m i R-1288$ expression changes were also noted for ESCCs with distant metastasis (70\%) and with poorly differentiated morphology (77\%)(Figure 2). Despite these associations, these correlations were not statistically significant (Table 1). Also, there was no association identified between the pattern of miR1288 expression and patients' age, gender as well as size and pathological stages of the cancer $(\mathrm{p}>0.05)$.

The mean follow-up period for the patients with ESCC was 1025 days (up to 6406 days). During the follow-up period, 46 patients survived, 27 died of cancer. The mean survival time of patients with high miR-1288 expression was 1854 days whereas that of low miR-1288 expression was 1186 days. This indicates a slightly better patient survival time of patients having ESCC with higher miR-1288 expression levels (Figure 3). However, the difference did not reach significant level $(\mathrm{p}>0.05)$. 


\subsection{Induced overexpression by miR-1288 mimic in oesophageal cancer cells (Figure 4)}

Expression of $m i R-1288$ was analysed at 24 and 48 hours of transfection in two oesophageal cancer cells (KYSE-520 and KYSE-70). In both cells, treatment with miR-1288 mimic (KYSE-520 $0^{+m i R-1288}$ and KYSE-70 $0^{+m i R-1288}$ ) showed remarkable overexpression of miR-1288 levels when compared to the control miRNA (KYSE-520 ${ }^{\text {miRScr }}$ and KYSE-70 ${ }^{\text {miRScr }}$ ) and non-transfected wild type (KYSE-520 ${ }^{\text {wildtype }}$ and KYSE-70 ${ }^{\text {wildtype }}$ ) cells. More than four thousand fold overexpression was noted in KYSE-520 ${ }^{+m i R-1288}$ cells with miR-1288 mimic treatment compared to that of HACAT cell line after 24 and 48 hours of transfection (Figure 3A). Similar over expression was also observed in KYSE-70 $0^{+m i R-1288}$ cells (Figure 4B). On the other hand, miR-1288 inhibitor has significantly reduced the miR-1288 expression levels in both ESCC cell groups (KYSE-520 $0^{+m i R-1288-I n}$ and KYSE-70 ${ }^{+m i R-1288-I n}$ ) at 24 and 48 hours of transfection (Figure 4A \& 4B).

\subsection{Increased proliferation of oesophageal cancer cells with miR-1288 transfection}

\section{(Figure 5)}

KYSE-520 $0^{+m i R-1288}$ and KYSE-70 ${ }^{+m i R-1288}$ cells showed increased cell proliferation when compared to control (KYSE-520 ${ }^{\mathrm{miRScr}}$ and KYSE-70 ${ }^{\mathrm{miRScr}}$ and non-transfected wild type (KYSE-520 $0^{\text {wildtype }}$ and KYSE-70 ${ }^{\text {wildtype }}$ ) counterparts. The increased proliferation of cells was noted in all three days of the experiment with a similar trend in both cell lines (Figure 5A \& B). In contrast, KYSE-520 ${ }^{\text {miR-1288-In }}$ and KYSE-70 ${ }^{+m i R-1288-I n}$ cells exhibited reduced cells proliferation compared to the both controls (scramble miRNA and wildtype) (Figure 5A \& B). 


\subsection{Higher clonogenic capacity of oesophageal cancer cells}

Transfection of oesophageal cancer cells with miR-1288 mimic (KYSE-520 ${ }^{+m i R-1288}$ and KYSE-70 ${ }^{+m i R-1288}$ ) showed remarkably increased colony formation properties in comparison to the control (KYSE-520 ${ }^{\mathrm{miRScr}}$ and KYSE-70 ${ }^{\mathrm{miRScr}}$ ) and non-transfected wild type oesophageal cancer (KYSE-520 ${ }^{\text {wildtype }}$ and KYSE-70 ${ }^{\text {wildtype }}$ ) cells (Figure 6). The number of colonies in KYSE-520 $0^{+m i R-1288}$ and KYSE-70 ${ }^{+m i R-1288}$ cells were significantly $(p<0.05)$ higher in comparison to the scramble miRNA treated and un-transfected cells.

\subsection{Increased penetration of BME-barrier of cancer cells with miR-1288 transfection}

Both ESCC cells overexpressed with miR-1288 (KYSE-520 ${ }^{+m i R-1288}$ and KYSE$70^{+m i R-1288}$ ) showed increased cell invasive properties. The relative fluorescence unit (RFU; which is proportional to the BME-barrier invading cells) from miR-1288 mimic treated cells was noticeably higher when compared to that of un-transfected and scramble control miRNA transfected cells (Figure 7). In KYSE-520+miR-1288 cells, RFU was $75 \pm 13$ whereas RFU from KYSE-520 ${ }^{\text {miRScr }}$ cell was $48 \pm 7$. Similar results (63 \pm 11 versus $45 \pm 7$ ) were also noted in case of KYSE-70 cells. Inhibitor of miR-1288 treatment reduced the invasive properties of ESCC cells (Figure 7).

\subsection{Manipulation of miR-1288 expression correlates with expression of FOXO1 tumour suppressor gene}

FOXO1 protein expression remarkably downregulated in miR-1288 mimic transfected (KYSE-520 ${ }^{+m i R-1288}$ and KYSE-70 ${ }^{+m i R-1288}$ ) cells when compared to their counter parts (Figure 8A). The relative protein band intensity of FOXO1 expressions in KYSE-70 ${ }^{\mathrm{miRScr}}$ and KYSE-520 ${ }^{\text {miRScr }}$ cells with scramble control miRNA transfection were $1.12 \pm 0.1$ and $1.13 \pm 0.05$ respectively (Figure $8 \mathrm{~B}$ ). On the other hand, the intensity of expression of FOXO1 
protein in miR-1288 mimics transfected cells (KYSE-520 $0^{+m i R-1288}$ and KYSE-70 ${ }^{+m i R-1288}$ ) were $0.47 \pm .12$ and $0.27 \pm 0.07$ respectively (Figure $8 B$ ). It was noted that in KYSE-520 ${ }^{+m i R-}$ 1288-In and KYSE-70 ${ }^{+m i R-1288-I n}$ cells FOXO1 protein expression was remarkably higher compared to the mimic treated cells (KYSE-520 ${ }^{+m i R-1288}$ and KYSE-70 ${ }^{+m i R-1288}$ ). The protein band intensity was $1.41 \pm 0.14$ vs $0.47 \pm .12$ and $0.83 \pm .11$ vs 0.27 for KYSE-520 and KYSE-70 cells respectively (Figure 8B \& C).

Expression of other tested proteins such as TAB3, p53, KRAS and BCL2 were noted to be unchanged (Figure 9). Downregulation of FOXO1 upon miR-1288 mimic transfection in oesophageal squamous carcinoma cell lines was further confirmed by immunofluorescence microscopy. FOXO1 protein was mainly located in the cytoplasm of the ESCC cells and minimally expressed in nucleus. Remarkable reduced expression of cytoplasmic FOXO1 protein was noted in ESCC cells treated with miR-1288 mimic when compared to that of control cells (Figure 9). This inverse modulation of FOXO1 protein was also noted with miR-1288 inhibition as the ESCC cells treated with miR-1288 inhibitor showed increased cytoplasmic FOXO1 protein expression under fluorescence microscope. 


\section{Discussion}

This study reported for the first time the altered expression levels of $m i R-1288$ in ESCCs tissues and cell lines. High levels of $m i R-1288$ expression in pathologically advanced ESCC tissues and its overexpression induced oncogenic properties in vitro by suppressing a key tumour suppressor marker FOXO1 suggest its regulatory roles in the pathogenesis of ESCC.

Downregulation or loss of expression of $m i R-1288$ was noted predominantly in colorectal carcinomas and ovarian carcinomas [9, 12]. Our recent study has reported miR1288 overexpression in 22\% (18/82) colorectal adenocarcinoma tissues and 78\% showed downregulation of miR-1288 expression [9]. The current study showed miR-1288 overexpression in 63\% (57/90) of the ESCC tissues and only 37\% of the ESCC tissues showed downregulation of $m i R-1288$. This difference in $m i R-1288$ could be attributed to the natural variation of $m i R-1288$ expression in various tissues. However, increased proliferation, colony formation and migration of ESCC cells in this study following miR1288 overexpression were consistent with the previous study findings [9]. Also, downregulation of endogenous $m i R-1288$ by its inhibitor decreased cell proliferation, clonogenic potential and invasive properties of ESCC cancer cells. Thus, these results indicate that the regulatory roles of $m i R-1288$ expression are tissue specific and its overexpression initiated the proliferation and progression of ESCCs.

Studies have reported the roles of microRNAs (miRNAs) in regulating various physiological, clinical, therapeutic, and prognostic aspects of human cancers [14-17]. In solid tumours, up regulation of miRNA expression is a common event and this expression is in turn associated with specific tissue types [18]. In ESCCs, overexpression of miRNAs has been reported to be a significant event in the regulation of carcinogenesis [19-21]. Kurashige et al. have reported overexpression of $m i R-223$ in ESCC of pathologically advanced stages 
and in patients with poor prognosis [20]. Similar to these results, the current study has also noted a predominant overexpression of $m i R-1288$ in ESCC patients with advanced pathological stages. On the other hand, patients with ESCC having overexpressed miR-1288 overexpressed ESCC exhibited a better survival rates. This opposite association noted in this study was non-significant, but analogues to our previous findings in colorectal adenocarcinomas [9]. Thus, $m i R-1288$ act as an oncogenic miRNA in the initiation and development of ESCC and its overexpression related better cancer survival might be attributed to its physiological interaction with various target proteins in the carcinogenesis. Additional studies with more cancer cases with longer follow-up period would be needed to confirm the predictive role of $m i R-1288$ in the prognosis of patients with ESCC.

Forkhead box $\mathrm{O}(F O X O)$ genes are involved in multiple signaling pathways in human cancers and play a critical role in carcinogenesis by regulating various physiological and pathological processes [22]. Activation of FOXO can control apoptotic pathways, cell proliferation, and cell cycle regulation [22]. FOXO1, a member of FOXO family, act as a tumor suppressor in cancers by inducing apoptosis, cell cycle arrest and oxidative and cellular stress [23]. Previous studies have reported that the 3' untranslated region of FOXO1 contains several potential miRNA binding sites and thus it can be regulated by several microRNAs [24-26]. Through informatics analysis using miRDB target prediction tool (http://mirdb.org/miRDB/), we found that there is a putative binding site in the $3^{\prime}$ untranslated region (UTR) of FOXO1 mRNA, to which miR-1288 seed sequence can bind [27]. This study has observed significant modulation of FOXO1 protein expression following the regulation of $m i R-1288$ expression (either activation or inhibition) (Figure 8). Simultaneously, the expression of TAB3 (a putative target of $m i R-1288$; http://mirdb.org/miRDB/) and some other growth related proteins such as p53, KRAS and BCL2 were not changed in different groups of cells (Figure 9). Also, suppression of 
cytoplasmic FOXO1 protein was noted predominantly in the ESCC cells while nuclear FOXO1 protein less distinct or minimally expressed in selected cell groups (Figure 9). In the nucleus, FOXO proteins act as transcriptional regulators, while in the cytoplasm, FOXO proteins get phosphorylated and undergo proteasomal degradation after ubiquitination without transcriptional modifications [28]. In addition, unphosphorylated FOXO1 can suppress tumor cell proliferation via regulation of p21, p27, and cyclin D1 gene transcription [28]. Thus, the miR-1288 induced post translational regulation and suppression of cytoplasmic FOXO1 could have a potential role in regulating esophageal cancer pathogenesis. Furthermore, miR-1288 may modulate the phosphorylation of FOXO1 protein and can further induce the oncogenic properties of ESCC cells by knocking down the tumor suppressor properties of FOXO1.

Our most recent study on miR-1288 has observed an important role of $m i R-1288$ overexpression in enhancing colon cancer cell proliferation [9]. Similar to the findings in colon cancer cells, this study also noted enhanced ESCC cell proliferation following activation of $m i R-1288$. In addition, other oncogenic in vitro features such as enhanced cell migration, increased colony formation and invasion properties were noted following miR1288 activation in ESCC cell lines [Figures 5-7]. Also, cancer inhibitory properties, such as reduced cellular proliferation, deceased colony formation and migration of cancer cells followed by downregulation of $m i R-1288$ further confirms the oncogenic features of $m i R$ 1288. These results can be attributed to the physiological mechanism behind the over expression of miR-1288 in pathologically advanced ESCC tissues. Also, slightly increased levels of miR-1288 expression in ESCC with metastasis (lymph node or distant) might be triggered by the activation of cancer cell invasion and migration by $m i R-1288$. Hence, the pathological aggressiveness of ESCC tumors in this study was well aligned with biological behavior of miR-1288 modulated viable cancer cells in vitro. Therefore it can be 
hypothesized that miR-1288 act as an oncogenic miRNA and plays a vital role in the pathogenesis of ESCCs by regulating various physiological and pathological processes in cancer cells. Future research is needed to understand the precise mechanism of action of this miRNA in vivo and its role in regulating other signalling pathways in ESCCs.

In summary, miR-1288 is frequently overexpressed in esophageal squamous cell carcinoma, and over expression of miR-1288 triggered activation of cell proliferation, colony formation and cell invasion in vitro of this cancer. These effects may be related to repression of the tumor suppressor protein, FOXO1 through negative regulation by miR-1288 at the post-translational level via binding the 3' untranslated region of FOXO1 mRNA. These findings imply that miR-1288 expression plays a key role in the molecular carcinogenesis of ESCCs and it also raises the possibility that anti-microRNA-1288 agents may have potential therapeutic value in patients with ESCC. 


\section{References}

1. Lam AK. Cellular and molecular biology of esophageal cancer. In: Esophageal cancer: prevention, diagnosis and therapy. Saba N F, El-Rayes B (Eds.), Springer, Chapter 2, 2015. pp. 25-40.

2. Lam AKY. Critical review: molecular biology of oesophageal squamous cell carcinoma. Crit Rev Oncol Hematol. 2000; 33: 71-90.

3. Lam KY, Ma L. Pathology of oesophageal cancers: local experience and current insights. Chin Med J. 1997; 110: 459-64.

4. Lam KY, Law S, Tung PH, Wong J. Esophageal basaloid squamous cell carcinoma: an unique clinicopathological entity with telomerase activity as a prognostic indicator. $\mathrm{J}$ Pathol. 2001; 195: 435-42.

5. Ebrahimi F, Gopalan V, Wahab R, Lu CT, Anthony Smith R, Lam AK. Deregulation of miR-126 expression in colorectal cancer pathogenesis and its clinical significance. Exp Cell Res. 2015; 339: 333-41.

6. Rahman MA, Salajegheh A, Smith RA, Lam AK. MicroRNA-126 suppresses proliferation of undifferentiated (BRAF(V600E) and BRAF(WT)) thyroid carcinoma through targeting PIK3R2 gene and repressing PI3K-AKT proliferation-survival signalling pathway. Exp Cell Res. 2015; 339: 342-50.

7. Salajegheh A, Vosgha H, Md Rahman A, Amin M, Smith RA, Lam AK. Modulatory role of miR-205 in angiogenesis and progression of thyroid cancer. J Mol Endocrinol. 2015; 55: 183-96.

8. Gopalan V, Smith RA, Lam AK. Downregulation of microRNA-498 in colorectal cancers and its cellular effects. Exp Cell Res. 2015; 330: 423-8. 
9. Gopalan V, Pillai S, Ebrahimi F, Salajegheh A, Lam TC, Le TK, et al. Regulation of microRNA-1288 in colorectal cancer: altered expression and its clinicopathological significance. Mol Carcinog. 2014; 53 Suppl 1: E36-44.

10. Amin M, Lam AK. Current perspectives of mi-RNA in oesophageal adenocarcinoma: Roles in predicting carcinogenesis, progression and values in clinical management. Exp Mol Pathol. 2015; 98: 411-8.

11. Slattery ML, Wolff E, Hoffman MD, Pellatt DF, Milash B, Wolff RK. MicroRNAs and colon and rectal cancer: differential expression by tumor location and subtype. Genes Chromosomes Cancer. 2011; 50: 196-206.

12. Gorringe KL, Ramakrishna M, Williams LH, Sridhar A, Boyle SE, Bearfoot JL, et al. Are there any more ovarian tumor suppressor genes? A new perspective using ultra high-resolution copy number and loss of heterozygosity analysis. Genes Chromosomes Cancer. 2009; 48: 931-42.

13. Bosman FT, Carneiro F, Hruban RH, Theise ND. Tumours of the oesophagus. In: WHO classification of tumours of the digestive system. WHO press, Lyon, 2010, Chapter 2, pp16-24.

14. Ebrahimi F, Gopalan V, Smith RA, Lam AK. miR-126 in human cancers: clinical roles and current perspectives. Exp Mol Pathol. 2014; 96: 98-107.

15. Vosgha H, Salajegheh A, Smith RA, Lam AK. The important roles of miR-205 in normal physiology, cancers and as a potential therapeutic target. Curr Cancer Drug Targets. 2014; 14: 621-37.

16. Maroof H, Salajegheh A, Smith RA, Lam AK. MicroRNA-34 family, mechanisms of action in cancer: a review. Curr Cancer Drug Targets. 2014; 14: 737-51.

17. Chruścik A, Lam AK. Clinical pathological impacts of microRNAs in papillary thyroid carcinoma: A crucial review. Exp Mol Pathol. 2015; 99: 393-8. 
18. Volinia S, Calin GA, Liu CG, Ambs S, Cimmino A, Petrocca F, et al. A microRNA expression signature of human solid tumors defines cancer gene targets. Proc Natl Acad Sci U S A. 2006; 103: 2257-61.

19. Hiyoshi Y, Kamohara H, Karashima R, Sato N, Imamura Y, Nagai Y, et al. MicroRNA-21 regulates the proliferation and invasion in esophageal squamous cell carcinoma. Clin Cancer Res. 2009; 15: 1915-22.

20. Kurashige J, Watanabe M, Iwatsuki M, Kinoshita K, Saito S, Hiyoshi Y, et al. Overexpression of microRNA-223 regulates the ubiquitin ligase FBXW7 in oesophageal squamous cell carcinoma. Br J Cancer. 2012; 106:182-8.

21. Kimura S, Naganuma S, Susuki D, Hirono Y, Yamaguchi A, Fujieda S, et al. Expression of microRNAs in squamous cell carcinoma of human head and neck and the esophagus: miR-205 and miR-21 are specific markers for HNSCC and ESCC. Oncol Rep. 2010; 23: 1625-33.

22. Fu Z, Tindall DJ. FOXOs, cancer and regulation of apoptosis. Oncogene. 2008; 27: 2312-9.

23. Myatt SS, Lam EW. The emerging roles of forkhead box (Fox) proteins in cancer. Nat Rev Cancer. 2007; 7: 847-59.

24. Hasseine LK, Hinault C, Lebrun P, Gautier N, Paul-Bellon R, Van Obberghen E. miR-139 impacts FoxO1 action by decreasing FoxO1 protein in mouse hepatocytes. Biochem Biophys Res Commun. 2009; 390: 1278-82.

25. Guttilla IK, White BA. Coordinate regulation of FOXO1 by miR-27a, miR-96, and miR-182 in breast cancer cells. J Biol Chem. 2009; 284: 23204-16.

26. Myatt SS, Wang J, Monteiro LJ, Christian M, Ho KK, Fusi L, et al. Definition of microRNAs that repress expression of the tumor suppressor gene FOXO1 in endometrial cancer. Cancer Res. 2010; 70: 367-77. 
27. Wong N, Wang X. miRDB: an online resource for microRNA target prediction and functional annotations. Nucleic Acids Res. 2015; 43 (Database issue): D146-52.

28. Wu L, Li H, Jia CY, Cheng W, Yu M, Peng M, et al. MicroRNA-223 regulates FOXO1 expression and cell proliferation. FEBS Lett. 2012; 586: 1038-43. 


\section{Figure legends}

Figure 1. Expression levels of $\mathbf{m i R}-1288$ in ESCC and normal cell lines. Both ESCC cell lines (KYSE-520 and KYSE-70) showed high expression of miR-1288 expression compared to non-cancer cell lines (HACAT. Inverse ratio of miR1288 versus RNU6B (control miRNA) [expression ratio] was used to represent the miRNA expression levels in different cell lines. Results are expressed as mean \pm standard deviation $(\mathrm{SD})(\mathrm{n}=3)$ and level of significance, *p $>0.05$ when compared to the HACAT cell line.

Figure 2. Histological grade of ESCC: The poorly-differentiated ESCC (2A) often had higher miR-1288 expression level than well-differentiated ESCC (2B)

Figure 3. The relationship between patients' survival and $\mathbf{m i R}-1288$ expression levels in ESCC: Patients with high miR-1288 expression levels in their colorectal cancer tissue had slightly better survival rates than patients with low miR-1288 levels. This difference do not reach statistical significance $(\mathrm{p}>0.05)$

\section{Figure 4. Overexpression of $\mathbf{m i R}-1288$ in ESCC cell lines following exogenous}

transfection of miR-1288 mimic. The miR-1288 expression levels in KYSE-520 (A) and KYSE-70 (B) cell lines. Significant difference in miRNA expression was noted in posttransfect cells with miR-1288 mimic. Expression levels were analysed in fold change and was compared with control cell lines (HACAT). A highest level of miR-1288 expression was noted after 24 hours of transfection and this duration was considered as the optimum for the downstream in vitro experiments. Treatment of cells (KYSE-520 and KYSE-70) with miR1288 inhibitor also remarkably reduced the expression of miR-1288 (A and B).

Figure 5. Cell proliferation assay for detecting miR-1288 induced cell proliferation. Results were plotted on different time intervals (days from 0 to 3) with mean value of absorbance. Both ESCC cells treated with miR-1288 mimic (KYSE-520+miR-1288 and KYSE$70^{+m i R 1288}$ ) showed increased cell proliferation when compared to control (KYSE-520 ${ }^{\text {miRScr }}$ 
and KYSE-70 ${ }^{\mathrm{miRScr}}$ ) and non-transfected wild type counterparts (KYSE-520 $0^{\text {wildtype }}$ and KYSE-70 ${ }^{\text {wildtype }}$ (A\&B). The increased proliferation of cells was noted in all three days for both cell lines. Cells treated with miR-1288 inhibitor (KYSE-520 ${ }^{+m i R-1288-I n}$ and KYSE$70^{+ \text {miR1288-In }}$ ) exhibited reduced proliferation compared to the counterparts (A\&B). Results are expressed as mean $\pm \mathrm{SD}(\mathrm{n}=3)$ and level of significance, ${ }^{* *} \mathrm{p}>0.01$ and ${ }^{*} \mathrm{p}>0.05$ when compared to control groups.

Figure 6. $\mathbf{m i R}-1288$ induced colony formation in ESCC cell lines. A: Overexpression of miR-1288 (KYSE-520 ${ }^{+m i R-1288}$ and KYSE-70 ${ }^{+m i R 1288}$ ) resulted in increased colony formation in both ESCC cell lines compared to control (KYSE-520 ${ }^{\text {miRScr }}$ and KYSE-70 ${ }^{\mathrm{miRScr}}$ ) and nontransfected (KYSE-520 ${ }^{\text {wildtype }}$ and KYSE-70 ${ }^{\text {wildtype }}$ ) cells. Inactivation of miR-1288 followed by inhibitor (KYSE-520 ${ }^{+m i R-1288-I n}$ and KYSE-70 ${ }^{+m i R 1288-I n}$ ) treatment reduced the clonogenic capacity of ESCC cells. B: Bar charts indicate that the number of cell colonies with miR1288 mimic were significantly $(\mathrm{p}=0.024)$ higher in comparison to control and un-transfected cells.

Figure 7. Enhanced cell invasion properties in miR-1288 overexpressed ESCC cells. Both KYSE-520 and KYSE-70 cells showed increased penetration of basement membrane extract (BME)-barrier following miR-1288 transfection. The BME-barrier invasion of cells was noted as relative fluorescence unit (RFU) and was significantly higher with miR-1288 over expression. A RFU of $75 \pm 13$ was noted in KYSE-520 $0^{+m i R-1288}$ cells compared to KYSE-

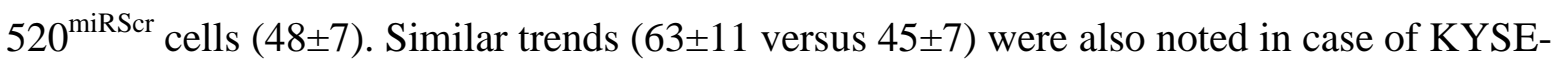
70 cells. Downregulation of miR-1288 with its inhibitor reduced the invasion ESCC cells. Results are expressed as mean $\pm \mathrm{SD}(\mathrm{n}=3)$ and level of significance, ${ }^{* *} \mathrm{p}>0.01$ and ${ }^{*} \mathrm{p}>0.05$ when compared to the control groups.

Figure 8: miR-1288 regulates the expression of FOXO1 tumour suppressor gene. A: FOXO1 protein expression was downregulated in miR-1288 transfected ESCC (KYSE- 
$520^{+m i R-1288}$ and KYSE-70 ${ }^{+m i R 1288}$ ) cells compared to un-transfected (KYSE-520 $0^{\text {wildtype }}$ and KYSE-70 ${ }^{\text {wildtype }}$ ) and scramble control miRNA transfected cells (KYSE-520 ${ }^{\text {miRScr }}$ and KYSE$\left.70^{\text {miRScr }}\right)$. The expression of FOXO1 protein was upregulated in KYSE-520 ${ }^{\text {miR-1288-In }}$ and KYSE-70 ${ }^{+m i R 1288-I n}$ cells compared to the counterparts. The miR-1288 mediated suppression of other proteins such as TAB3, p53, KRAS and BCL2 was noted to unaltered in all the ESCC cell groups. B: The relative protein band intensity of FOXO1 in KYSE-520 $0^{+m i R-1288}$ cells was noted to be $0.47 \pm .12$ (versus $1.12 \pm 0.1$ ). The protein band intensity of KYSE$520^{\text {+miR-1288-In }} 1.41 \pm 0.14$ C): The intensity of FOXO1 protein band in KYSE-70 ${ }^{+m i R-1288}$ was $0.27 \pm 0.07$ (versus $1.13 \pm 0.05$ ) whereas in KYSE-70 ${ }^{+m i R-1288-I n}$ cells the protein band intensity

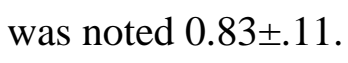

Figure 9: Confirmation of miR-1288 mediated downregulation of FOXO1 protein via immunofluorescence microscopy. FOXO1 protein was predominantly expressed in the cytoplasm of ESCC cells. Similar to Western blot findings, reduced expression of FOXO1 protein was noted in both ESCC cell lines treated with miR-1288 compared to those of untransfected and scramble control miRNA transfected cells. Also, miR-1288 inactivation by inhibitor treatment increased the expression of FOXO1 protein in ESCC cells. 
Table 1. Expression pattern of miR-1288 and its correlation with clinicopathological features of ESCC

\begin{tabular}{|c|c|c|c|c|}
\hline Type & No & High Expression & Low Expression & $\mathrm{p}$ value \\
\hline \multicolumn{5}{|l|}{ Gender } \\
\hline Male & 77 (85.6\%) & $48(62.3 \%)$ & 29 (37.7\%) & \multirow[t]{2}{*}{0.442} \\
\hline Female & $13(14.4 \%)$ & $9(69.2 \%)$ & $4(30.8 \%)$ & \\
\hline \multicolumn{5}{|l|}{ Age } \\
\hline$<60$ & 31 (34.4\%) & $23(74.2 \%)$ & $8(25.8 \%)$ & \multirow[t]{2}{*}{0.092} \\
\hline$>60$ & $59(65.6 \%)$ & $34(57.6 \%)$ & $25(42.4 \%)$ & \\
\hline \multicolumn{5}{|l|}{ Site } \\
\hline Lower & $36(40 \%)$ & $21(58.3 \%)$ & 15 (41.7\%) & \multirow{3}{*}{0.703} \\
\hline Middle & 44 (48.9\%) & 29 (65.9\%) & 15 (34.1\%) & \\
\hline Upper & $10(11.1 \%)$ & 7 (70\%) & $3(30 \%)$ & \\
\hline \multicolumn{5}{|l|}{ Size } \\
\hline$<50 \mathrm{~mm}$ & $56(62.2 \%)$ & 35 (62.5\%) & 21 (37.5\%) & \multirow[t]{2}{*}{0.508} \\
\hline$>50 \mathrm{~mm}$ & 34 (37.8\%) & 22 (64.7\%) & 12 (35.3\%) & \\
\hline \multicolumn{5}{|c|}{ Histological grade } \\
\hline Well & $32(35.6 \%)$ & 18 (56.3\%) & $14(43.8 \%)$ & \multirow[t]{3}{*}{0.376} \\
\hline Moderate & $41(45.6 \%)$ & $26(63.4 \%)$ & 15 (36.6\%) & \\
\hline Poor & 17 (18.9\%) & $13(76.5 \%)$ & $4(23.5 \%)$ & \\
\hline \multicolumn{5}{|l|}{ T staging } \\
\hline T1\&T2 & $6(6.7 \%)$ & $3(50 \%)$ & $3(50 \%)$ & \multirow[t]{2}{*}{0.385} \\
\hline T3\&T4 & 84 (93.3\%) & $54(64.3 \%)$ & 30 (35.7\%) & \\
\hline \multicolumn{5}{|c|}{ Lymph node metastasis } \\
\hline Present & 70 (77.8\%) & 45 (64.3\%) & 25 (81.8\%) & \multirow[t]{2}{*}{0.460} \\
\hline Absent & $20(22.2 \%)$ & 12 (60\%) & $8(40 \%)$ & \\
\hline \multicolumn{5}{|c|}{ Distant metastasis } \\
\hline Present & 10 (11.1\%) & 7 (70\%) & $3(30 \%)$ & \multirow[t]{2}{*}{0.464} \\
\hline Absent & $80(88.9 \%)$ & $50(62.5 \%)$ & 30 (37.5\%) & \\
\hline \multicolumn{5}{|c|}{ Pathological staging } \\
\hline I \&II & 24 (26.7\%) & $16(66.7 \%)$ & $8(33.3 \%)$ & \multirow{2}{*}{0.445} \\
\hline III \& IV & $66(73.3 \%)$ & $41(62.7 \%)$ & $25(37.9 \%)$ & \\
\hline
\end{tabular}

